\title{
Prevalence and duration of gastrointestinal symptoms before diagnosis of Inflammatory Bowel Disease and predictors of timely specialist review: a population-based study
}

\section{J. Blackwell ${ }^{1}$, S. Saxena ${ }^{2}$, N. Jayasooriya ${ }^{1}$, A. Bottle ${ }^{2}$, I. Petersen ${ }^{3,4}$, M.Hotopf ${ }^{5,6}$, C. Alexakis ${ }^{1}$, R.C. Pollok ${ }^{1}$, POP-IBD study group}

${ }^{1}$ Dept. Gastroenterology, St George's Healthcare NHS Trust

*St George's University London, UK

${ }^{2}$ School of Public Health, Imperial College London, London, UK

${ }^{3}$ Dept. Primary Care and Population Health, University College London, London, UK

${ }^{4}$ Dept. Clinical Epidemiology, Aarhus University, Denmark

${ }^{5}$ Institute of Psychiatry, Kings College London, London, UK

${ }^{6}$ South London and Maudsley NHS Foundation Trust, London, UK

\section{Ethical approval:}

ISAC Protocol number: 15_018R

\section{Contributorship}

The POP-IBD study group is a collaboration between St George's, University of London, Imperial College London, University College London and King's College London, conducting population based studies in the field of Inflammatory Bowel Disease. JB and SS contributed equally to this project and are joint first authors. JB, SS, NJ, RP, CA, IP, \& MH conceived and designed this study. JB and SS prepared the data and carried out statistical analysis overseen by IP and $A B$. All authors contributed to the development of the analysis, interpreting data and preparing the manuscript. RP will act as the guarantor for the article.

\section{Competing interests}

None declared

\section{Provenance}

Not commissioned

\section{Acknowledgements}

None 


\section{Funding and disclaimer:}

This work was supported by the Living with IBD Research Programme at Crohn's \& Colitis UK (grant number: SP2018/3). This funding source had no role in the design or execution of this study or in the analysis and interpretation of the data. The views expressed are those of the authors and not necessarily those of Crohn's \& Colitis UK.

RP received support by a Wellcome Trust Institute Strategic Support Fund (ISSF) grant. $\mathrm{MH}$ acknowledges support from the National Institute of Health Research Biomedical Research Centre at the Maudsley, and is an NIHR Senior Investigator.

SS is funded by the National Institute for Health Research (NIHR) School for Public Health Research (SPHR) and NIHR Northwest London Applied Research Collaboration (ARC). The School for Public Health Imperial College London is also grateful for support from the Imperial NIHR Biomedical Research Centre.

The Dr Foster Unit at Imperial is affiliated with the National Institute of Health Research (NIHR) Imperial Patient Safety Translational Research Centre. The NIHR Imperial Patient Safety Translational Centre is a partnership between the Imperial College Healthcare NHS Trust and Imperial College London. The Dr Foster Unit at Imperial College are grateful for support from the NIHR Biomedical Research Centre funding scheme.

The views expressed in this publication are those of the authors and not necessarily those of the NHS, the NIHR or the Department of Health

\section{ORCID}

$J B$

0000-0003-4278-3720

SS

0000-0003-3787-2083

NJ $\quad 0000-0003-1343-2446$

AB $\quad 0000-0001-9978-2011$

IP $\quad 0000-0002-0037-7524$

$\mathrm{MH} \quad$ 0000-0002-3980-4466

CA $\quad 0000-0002-5487-0752$

RP 0000-0001-6452-6763

\section{Conflict of interest:}

None declared. 


\section{Abstract}

\section{Background and Aims}

Lack of timely referral and significant waits for specialist review amongst individuals with unresolved gastrointestinal (GI) symptoms can result in delayed diagnosis of Inflammatory Bowel Disease (IBD). Aims: To determine the frequency and duration of GI symptoms and predictors of timely specialist review before the diagnosis of both Crohn's Disease (CD) and ulcerative colitis (UC).

\section{Methods}

Case-control study of IBD matched 1:4 for age and sex to controls without IBD using the Clinical Practice Research Datalink from 1998-2016.

\section{Results}

We identified 19,555 cases of IBD, and 78,114 controls. 1 in 4 cases of IBD reported gastrointestinal symptoms to their primary care physician more than 6 months before receiving a diagnosis. There is a significant excess prevalence of GI symptoms in each of the 10 years before IBD diagnosis. GI symptoms were reported by $9.6 \%$ and $10.4 \%$ at 5 years before CD and UC diagnosis respectively compared to $5.8 \%$ of controls. Amongst patients later diagnosed with IBD, $<50 \%$ received specialist review within 18 months from presenting with chronic Gl symptoms. Patients with a previous diagnosis of irritable bowel syndrome or depression were less likely to receive timely specialist review (IBS: $\mathrm{HR}=0.77,95 \% \mathrm{Cl} 0.60-0.99$, depression: $\mathrm{HR}=0.77,95 \% \mathrm{Cl} 0.60-0.98)$.

\section{Conclusions}

There is an excess of GI symptoms 5 years before diagnosis of IBD compared to the background population which are likely attributable to undiagnosed disease. Previous diagnoses of IBS and depression are associated with delays in specialist review. Enhanced pathways are needed to accelerate specialist referral and timely IBD diagnosis.

\section{Key words:}

Inflammatory bowel disease, Crohn's disease, Ulcerative colitis, Chronic diarrhoea, Abdominal pain, Gastro-intestinal symptoms, Irritable bowel syndrome, depression, diagnosis, delayed diagnosis 


\section{Introduction}

Timely diagnosis of Inflammatory Bowel Disease (IBD) is essential to enable early treatment to alleviate symptoms and prevent disease progression. Individuals who wait for more than 2 years from the onset of gastrointestinal symptoms to Crohn's disease (CD) diagnosis are at increased risk of emergency surgery. ${ }^{1}$ Likewise, those who are symptomatic for more than 6 months prior to their diagnosis of ulcerative colitis (UC) are more likely to undergo colectomy. ${ }^{2}$ Early treatment with immunomodulators and biologics can reduce the risk of disease progression to stricturing (B2) and penetrating (B3) disease phenotype and the consequent need for intestinal and perianal surgery in CD by more than $50 \% \cdot{ }^{3-6}$ In UC, early escalation of medical therapy has been shown to improve quality of life, although its impact on requirement for surgery is not fully established. ${ }^{4,7-11}$ Prompt diagnosis can be challenging since symptoms of $C D$ and $U C$ including abdominal pain, diarrhoea or rectal bleeding, also occur in other more common conditions such as irritable bowel syndrome (IBS) or haemorrhoids which can make diagnosis difficult. ${ }^{12}$ One in six individuals with IBD initially receive a diagnosis of irritable bowel syndrome ${ }^{13}$ and one in seven individuals with UC are initially diagnosed with haemorrhoids. ${ }^{14}$ This can result in a delayed specialist referral and diagnosis and a consequent missed opportunity to commence early treatment to prevent disease progression. Previous studies report the median duration of symptoms before diagnosis to be between 6-9 months for $C D$ and 2-7 months for UC, with a quarter of individuals waiting more than a year. ${ }^{2,14,15}$ However, these studies do not account for the baseline prevalence of gastrointestinal symptoms in the general population and may overestimate the duration and prevalence of symptoms attributable to undiagnosed IBD.

Guidelines recommend patients experiencing gastrointestinal symptoms for more than 6 weeks should be referred to a gastroenterologist for timely specialist review within 4 weeks but it is unclear whether this is mirrored in real-world clinical practice. ${ }^{16,17}$

We designed a nationally representative case control study to examine the duration of symptoms before IBD diagnosis. Further to this we performed a linked analysis to determine the timeliness of 
specialist referral and the influencing factors like prior diagnosis of Irritable bowel syndrome and psychiatric co-morbidity. The aim of our study was firstly to examine prevalence of gastrointestinal symptoms attributable to undiagnosed IBD before diagnosis and second identify predictors for timely specialist review.

\section{Methods}

\section{Data source}

Using the Clinical Practice Research Datalink (CPRD) we identified all cases of CD and UC diagnosed between January 1st 1998 and May 1st 2016. CPRD is one of the largest validated primary care research databases in the world. It contains nationally representative, longitudinal, patient-level, anonymised electronic health records of 13 million patients from more than 700 general practices and is broadly representative of the United Kingdom (UK) population. The median follow-up for individuals registered on CPRD is 9.4 years, allowing study of long-term outcomes. ${ }^{18}$ Primary care physicians use Read codes to record symptoms, signs and diagnoses. Data are audited to ensure accuracy and completeness. Participating practices need to achieve and maintain 'Up to standard' status to continue contributing to the dataset. The database's primary purpose is for epidemiological research and the coding system has been previously validated for use in IBD. ${ }^{19}$ Numerous IBD related studies have been undertaken using it. ${ }^{20,21}$

Data were linked to the Hospital Episodes Statistics Outpatient database (HES OP) where available. HES OP contains details of all outpatient appointments in all NHS hospitals in England. We obtained ethical and scientific approval for the use of CPRD and HES for our study from the Independent Scientific Advisory Committee (ISAC Protocol number: 15_018R). 


\section{Incident case definition}

We defined incident IBD cases as individuals with a first ever diagnosis Read code for either CD or UC at least one year after registering with an 'Up To Standard' practice for the period January 1st 1998 to May 1 st 2016 in accordance with published and validated methods. ${ }^{19,22,23}$

We excluded individuals if they had codes for both CD and UC, or indeterminate codes (e.g. 'nonspecific colitis'). Individuals were followed-up from their date of registration on the database until the date of their IBD diagnosis, defined as the date of the first recorded code for an IBD diagnosis in CPRD.

To examine predictors of receiving timely specialist review we identified all cases with chronic GI symptoms ( 2 consultations within a 6 month period at least 6 weeks apart, Appendix 1 ). We identified all individuals with HES outpatient data and at least 10 years registration with their primary care practice before IBD was diagnosed. Individuals whose first presentation with chronic GI symptoms occurred before $01 / 01 / 2003$ were excluded from this analysis as HES outpatient data was not collected before this point. 


\section{Control Groups}

We identified separate control groups for CD and UC cases from CPRD to establish the annual prevalence of Gl symptoms in the general population without IBD. The CD and UC cases were matched 1:4 to controls according to sex and age, defined by year of birth. Individuals were eligible to be controls if they had no recorded diagnosis of IBD at any stage of their follow-up. After stratification by age and sex, controls were selected at random. Controls were each assigned the IBD diagnosis date of their matched IBD individual as a pseudo-diagnosis. ${ }^{13}$

\section{Excess prevalence of GI symptoms before IBD diagnosis}

We measured the period prevalence of GI symptoms in each of the 10 years before IBD diagnosis. Through a consensus process, a clinical steering group of clinicians with an interest in IBD developed lists of Read codes to identify symptoms of IBD, including abdominal or perianal pain, diarrhoea and rectal bleeding (Appendix 2 - Code List). Individuals were considered to have prevalent GI symptoms in a given year if their primary care physician recorded at least one code for Gl symptoms in their medical records in that year. We included individuals in the denominator for each year if they were registered with their primary care practice that entire year. We calculated the excess prevalence of GI symptoms attributable to IBD in each of the 10 years before IBD diagnosis as the prevalence of GI symptoms in the $\mathrm{CD}$ and $\mathrm{UC}$ cohorts minus the baseline prevalence of $\mathrm{Gl}$ symptoms in their respective control groups. To avoid recording Gl symptoms reported within six months of the diagnosis of IBD we defined the first year before IBD diagnosis as the period eighteen to six months before the date of the first recorded diagnosis of IBD in CPRD. 


\section{Time to specialist review}

We identified all individuals with IBD who had presented to a primary care physician with chronic GI symptoms lasting more than 6 weeks prior to their diagnosis. We measured the time from presentation in primary care to specialist review. The date of presentation with chronic Gl symptoms was defined as the date of the second primary care physician consultation for Gl symptoms. We defined first specialist review as the date of the first outpatient appointment recorded in the HES OP database with a gastroenterologist, paediatric gastroenterologist or colorectal surgeon.

We examined potential predictors for timely specialist review. These were sex, age at presentation, socio-economic status (index of multiple deprivation), smoking status, prior diagnosis of IBS, prior diagnosis of depression, unattended appointments and era of presentation. Older age, smoking and low socioeconomic status have been associated with diagnostic delay of other chronic conditions. ${ }^{24-}$ 27 We defined individuals as 'smokers', 'ex-smokers' or 'non-smokers' based on codes for smoking status in the 10 years before presentation with chronic Gl symptoms. Individuals whose most recent code indicated active smoking were classed as 'smokers' and those with codes indicating previous but not current smoking were classed as 'ex-smokers'; individuals with only 'non-smoker' codes were classed as 'non-smokers'. Individuals without data on smoking have previously been shown to likely be either 'never-smokers' or 'non-recent smokers' and were therefore classed as 'nonsmokers' ${ }^{28}$

We used the Index of Multiple Deprivation (IMD) a postcode-linked measure of socio-economic deprivation to assign individuals to 1 of 5 groups using IMD quintiles, from IMD group 1 (least deprived) to 5 (most deprived)

We identified any individuals who had codes for IBS, depression or depressive symptoms before presentation with chronic Gl symptoms. ${ }^{29}$ We identified unattended specialist appointments 
between presentation with chronic Gl symptoms and the first attended specialist appointment, to establish the impact of this on an individual's chances of receiving specialist review.

To examine changes in physicians' diagnostic practice over the study period, we grouped individuals according to their era of presentation (i.e. 2003-2006, 2007-2010, 2011-2013, 2014-2016).

\section{Statistical Analysis}

We tabulated the prevalence of GI symptoms in each of the ten years leading up to IBD diagnosis among cases of $C D$ and $U C$ and their respective control groups. We estimated the risk difference of Gl symptoms, with $95 \%$ confidence intervals, between cases and controls.

We used Cox regression to determine hazard ratios (HR) for specialist review following presentation with Gl symptoms and identified predictors of specialist review in cases who ultimately received a diagnosis of IBD. In this model we adjusted for all variables listed in the "Time to Specialist Review" section.

We used Kaplan-Meier analysis to present the time to first specialist review following presentation with chronic Gl symptoms.

We conducted a sensitivity analysis, to include any outpatient appointments coded only as 'general internal medicine' or 'general surgery', in case some gastroenterology and colorectal surgery appointments were not specifically coded. This allowed us to estimate the highest possible proportion of patients seen by a specialist in secondary care in the 18 months following presentation.

All analyses were performed using STATA 15 (Statacorp LP, College Station, TX, USA). 


\section{Results}

Between January 1st, 1998 and May 1st, 2016 we identified 5,874 incident cases of CD and 13,681 incident cases of UC and 78,114 age and sex matched controls (Table 1).

\section{Excess prevalence of gastrointestinal symptoms before IBD diagnosis}

We found individuals with $C D$ and UC were four times more likely to visit their primary care physician for $\mathrm{Gl}$ symptoms than their respective control groups between eighteen and six months before diagnosis (CD: $29.1 \%$ vs. $6.5 \%$, risk difference $22.6 \%, 95 \% \mathrm{Cl} 21.3 \%-23.9 \%$, UC: $23.9 \%$ vs $6.7 \%$, risk difference $17.2 \%, 95 \% \mathrm{Cl} 16.4 \%-18.0 \%$, Figures 1 and 2).

There were higher proportions of abdominal or perianal pain $(16.8 \%$ vs. $4.0 \%$, risk difference $12.8 \%$, $95 \% \mathrm{Cl} 11.7 \%-13.8 \%$ ), diarrhoea (12.2\% vs. $1.8 \%$, risk difference $10.4 \%, 95 \% \mathrm{Cl} 9.5 \%-11.3 \%$ ) and rectal bleeding ( $4.5 \%$ vs. $1.1 \%$, risk difference $3.4 \%, 95 \% \mathrm{Cl} 2.8 \%-4.0 \%$ ) among cases of $\mathrm{CD}$ compared with their control group during the period eighteen to six months before diagnosis (Figure 1). Similarly the UC cohort had more rectal bleeding ( $10.6 \%$ vs. $1.2 \%$, risk difference $9.3 \%, 95 \% \mathrm{Cl} 8.8 \%$ 9.9\%), diarrhoea ( $8.4 \%$ vs. $2.3 \%$, risk difference $6.7 \%, 95 \% \mathrm{Cl} 6.2 \%-7.2 \%$ ) and abdominal or perianal pain (8.5\% vs $3.9 \%$, risk difference $4.6 \%, 95 \% \mathrm{Cl} 4.0 \%-5.1 \%$ ) during the same period (Figure 2 ).

Assuming the excess $\mathrm{Gl}$ symptoms were attributable to undiagnosed IBD we estimated $22.6 \%$ of $C D$ and $17.1 \%$ of UC individuals were already symptomatic from undiagnosed IBD in the period eighteen to six months before diagnosis.

In the period five years before diagnosis (5.5-4.5 years before diagnosis): $10.4 \%$ of CD individuals reported $\mathrm{Gl}$ symptoms, an excess of $4.2 \%$ relative their control group (95\% $\mathrm{Cl} 3.2 \%-5.2 \%$ ). In the same period $9.6 \%$ of UC individuals reported GI symptoms, an excess of $4.0 \%$ relative to their control group (95\% $\mathrm{Cl} 3.4 \%-4.6 \%$ ). Likewise we found a significant excess of $\mathrm{Gl}$ symptoms amongst patients 
who go on to develop IBD in each of the ten years prior to their diagnosis compared to matched controls (Appendices 3 and 4 - Prevalence of GI symptoms before IBD diagnosis).

\section{Time to specialist review}

We identified 1,034 individuals who had presented to their primary care physician with chronic GI symptoms before diagnosis with IBD (Table 2 and Appendix 1).

Of these $5.6 \%(n=58)$ received specialist review within 4 weeks, $32 \%(n=329)$ within 6 months and $50 \%(n=513)$ within 18 months.

A further 273 individuals with chronic Gl symptoms had a record of a 'general internal medicine' or 'general surgery' outpatient appointment but no appointment that met our stricter definition of specialist review. We included these individuals and those in the main analysis within a sensitivity analysis, where any general internal medicine or general surgery appointments were also considered specialist review. Using this wider definition, rates of specialist review rose to $23 \%$ at 4 weeks $(n=300), 61 \%$ at 6 months ( $n=796)$ and $74 \%$ at 18 months $(n=964)$.

Compared with individuals presenting with chronic Gl symptoms in the era 2003-2006 those presenting in the era 2014-2016 were significantly more likely to receive specialist review within 4 weeks ( $2.1 \%$ vs. $15.2 \%), 6$ months ( $17.6 \%$ vs. $75.8 \%$ ) and 18 months ( $32.6 \%$ vs. $100 \%$, Figure 3 ).

Individuals with a previous diagnosis of IBS or depression were less likely to receive timely specialist review than those without (IBS: $\mathrm{HR} 0.77,95 \% \mathrm{Cl}$ 0.60-0.99, Figure 4, Depression: $\mathrm{HR}=0.77,95 \% \mathrm{Cl}$ 0.60-0.98, Table 2).

Fourteen per cent ( $n=142$ ) of patients presenting with chronic Gl symptoms were referred for specialist review but did not attend their appointment. These were $32 \%$ less likely to receive specialist review within 18 months (Table 2). The risk of missed appointments was similar regardless 
of sex, age at presentation, smoking status, socioeconomic status or a previous diagnosis of depression or IBS.

\section{Discussion}

\section{Main findings}

This is the largest study to date to examine the prevalence of GI symptoms in the decade before IBD diagnosis compared with matched control groups drawn from the general population. There is a significant excess of Gl symptoms amongst patients who go on to develop IBD in each of the ten years prior to their diagnosis compared to matched controls. We found one in ten individuals visited their primary care physician for gastrointestinal symptoms 5 years before being diagnosed with either CD or UC, compared with one in twenty individuals in the matched control group. Only $5.6 \%$ of individuals with IBD received timely specialist review within 4 weeks of presenting to their primary care physician with chronic Gl symptoms, although this rose to $15.2 \%$ in the most recent era of our study (2014-2016). Individuals with either a premorbid diagnosis of IBS or depression were less likely to receive timely specialist review after presenting to their primary care physician with chronic Gl symptoms.

\section{Findings in relation to previous studies}

The time from the onset of GI symptoms to IBD diagnosis has generally been reported to be longer for CD than UC. ${ }^{15,30,31}$ In contrast, our study found the prevalence of GI symptoms in the years before diagnosis of UC was similar to that of CD. In a study of the Swiss IBD Cohort $10 \%$ of patients reported first presenting to a physician with Gl symptoms two years before being diagnosed with UC. ${ }^{15}$ We found even five years before the diagnosis of UC, one in ten patients were already visiting their primary care physician with GI symptoms. This may be because we used prospectively collected data as opposed to questionnaires that are subject to recall bias. It is also possible UC patients in the 
Swiss study considered only rectal bleeding as a symptom of UC. However, in our study, diarrhoea and abdominal pain comprised more than half of the excess GI symptoms in the UC cohort 2-10 years before diagnosis. This suggests these symptoms, which are common after diagnosis, may also in some individuals precede rectal bleeding in the natural history of UC. ${ }^{32}$

Our study revealed that each year between $4-6 \%$ of primary care patients, who do not have a subsequent diagnosis of IBD, present to their primary care physician with GI symptoms. Like the Swiss cohort study, we found $10 \%$ of CD individuals reported Gl symptoms five years before IBD diagnosis, but in the current study $6 \%$ of the control group reported GI symptoms in that same period, leaving an excess of $4 \%$. By establishing the excess prevalence of $\mathrm{Gl}$ symptoms we obtained a more accurate estimate of the prevalence of GI symptoms likely attributable to undiagnosed IBD.

UK IBD standards state individuals with suspected IBD should receive specialist review within four weeks. ${ }^{17}$ This is the first study to examine whether this national standard is being met. We found only $5.6 \%$ of individuals with chronic Gl symptoms received specialist review within four weeks. Encouragingly, in more recent era there has been a significant and progressive improvement in the rates of specialist review after presentation with chronic GI symptoms (Figure 3), perhaps reflecting improved diagnostic pathways with the increased use of surrogate biomarkers such as faecal calprotectin as an aid to risk stratification and diagnosis in the latter era of this study. ${ }^{33,34}$

ECCO guidelines state that to avoid diagnostic delay clinicians should consider investigating chronic non-specific symptoms which may mimic IBS. ${ }^{35}$ Interestingly, our study found a previous diagnosis of IBS was associated with a reduced likelihood of specialist review (Figure 4), possibly due to clinicians assuming IBS to be the cause of the patient's symptoms. ${ }^{36}$ Likewise, we found a previous diagnosis of depression was associated with a relative delay in specialist review. It is possible primary care physicians may consider GI symptoms more likely to be functional in this group and hold off onward referral. ${ }^{37,38}$ An alternative explanation may be that individuals with depression 
have different health-seeking behaviours and are less likely to request a referral to a specialist or attend appointments, although adjusting for unattended appointments did not alter our findings. ${ }^{39}$

\section{Strengths and Limitations}

To our knowledge this is the largest study to examine the prevalence of Gl symptoms in the 10 years before IBD diagnosis. Its size makes it highly unlikely that our results are due to chance. Data were drawn from a large nationally representative validated primary care research database linked to a national secondary care data source free of referral centre and recall bias, compared with previous studies which relied on retrospective questionnaires. In the UK universal healthcare coverage is provided by the National Health Service (NHS). It requires all individuals to be registered with a primary care physician, enabling high quality representative population-based studies using primary care data. In contrast with previous studies we used an age and sex matched control group without IBD to determine the baseline prevalence of GI symptoms among people who did not later receive a diagnosis of IBD during their follow-up. This allowed us to examine what proportion of Gl symptoms is likely to be attributable to IBD.

We captured data on primary care physician consultations for Gl symptoms. A previous study has reported 1 in 10 patients with IBD wait more than a year from the time of onset of their symptoms before first visiting a doctor which is a potential limitation to the study. ${ }^{15}$ This is more likely to affect males who are less likely to consult a primary care physician, even after adjusting for equivalent morbidity..$^{40}$ Individuals may have been diagnosed with IBD directly during an emergency hospital admission and never visited their primary care physician regarding GI symptoms before this. Coding of Gl symptoms may have been incomplete as we were unable to capture data recorded as free-text, meaning the burden of diagnostic delay may be even greater than we report. We were unable to capture data on faecal calprotectin as this test only became available toward the end of our study period and it is not consistently recorded in CPRD. ${ }^{12}$ 
We used a strict definition of specialist review, which only included hospital appointments coded as gastroenterology, paediatric gastroenterology or colorectal surgery. However some appointments may not have been coded as a specific specialty and would not have been captured in our definition. This was particularly likely to affect our first era, 2003-2006, when $16 \%$ of all HES OP appointments were coded only as general internal medicine or surgery, although by 2016 this affected only $1 \%$ of appointments. We therefore conducted a sensitivity analysis using a broader definition of specialist review to include any outpatient appointments which had not been coded as a specific specialty. Incomplete coding partially explains the higher rates of specialist review seen in the more recent era relative to 2003-2007, but our sensitivity analysis using the wider definition of specialist review still demonstrated a significant improvement in rates of specialist review in the more recent era (Appendix 5).

Some individuals may have been reviewed in the private healthcare sector and this would not have been captured in our study. It may also be possible some patients were referred directly for colonoscopy on a suspected cancer referral pathway, which was also not captured in our study. It is therefore possible that for a small proportion of cases the time to specialist review may have been overestimated.

Despite our study design using matched control groups, some residual confounding remains. For example, we did not have data on severity of Gl symptoms which may have also influenced the time to specialist review.

\section{Implications}

Our findings highlight a pressing need to improve the timely detection of IBD among symptomatic patients consulting in primary care. Even in the UK, where $98 \%$ of patients have access to universal coverage of healthcare, one in twenty-five individuals who are eventually diagnosed with IBD have GI symptoms for at least 5 years before diagnosis. Diagnostic delay of this magnitude is likely to negatively impact quality of life for these patients, ${ }^{41}$ creating uncertainty about the possible cause of 
symptoms as well as leaving pain, troublesome diarrhoea and rectal bleeding untreated. A Danish register-based study demonstrated individuals with IBD had significantly higher health costs in each of the 10 years before diagnosis relative to controls. ${ }^{42}$

Individuals with IBD were more likely to use health services but also had lower labour production value due to periods of unemployment. This may be a consequence of the excess GI symptoms identified during the pre-diagnostic period in our study and timely diagnosis of IBD may reduce the cost burden associated with diagnostic delay. Furthermore, several studies have indicated diagnostic delay is associated with disease progression, increased steroid use and an increased risk of surgery in both $C D$ and $U C .{ }^{1,2,31}$ Early initiation of therapy may improve the natural history of $C D$, delaying or preventing progression to stricturing (B2) or penetrating (B3) phenotype, although an equivalent disease modifying effect is yet to be demonstrated in UC. ${ }^{4-6,11,43,44}$ Even so, timely diagnosis of UC remains a crucial goal as treatment significantly improves symptoms and patients' quality of life. ${ }^{8}$

Clinicians need a higher index of suspicion for IBD and fast-track referral pathways should be implemented. Less than a tenth of individuals with chronic Gl symptoms receive timely specialist review within the recommended 4 weeks. ${ }^{16,17}$ Although this improved significantly in the more recent era of our study this trend is likely to have reversed during the COVID-19 pandemic which has resulted in substantially higher thresholds for investigation and inevitable delay in referral from primary care for all but the sickest. After the pandemic recedes it is probable there will remain a prolonged backlog in services which must be addressed carefully in planning future diagnostic pathways.

An IOIBD initiative developed a red flag index for early diagnosis of Crohn's Disease in patients presenting with $\mathrm{Gl}$ symptoms. ${ }^{34} \mathrm{~A}$ validation study found the index, based on history alone, had only $50 \%$ and $58 \%$ sensitivity and specificity respectively, meaning many diagnoses of CD would be missed. ${ }^{45}$ However when used in conjunction with faecal calprotectin, a non-invasive biomarker of intestinal inflammation, the sensitivity and specificity rose to $100 \%$ and $72 \%$ respectively. The BSG 
guidelines recommend any individuals presenting with $\mathrm{Gl}$ symptoms lasting more than 4 weeks, where it is unclear whether further investigation is warranted, should have a faecal calprotectin tested and if this is raised further investigation is appropriate. ${ }^{16}$

Clinicians need to be mindful that diagnostic labels of IBS and depression are associated with a relatively increased delay in specialist review for GI symptoms. Raising public awareness of the importance of reporting unresolved GI symptoms to primary care physicians may facilitate timely diagnosis. ${ }^{46}$ The Royal College of General Practitioners and Crohn's and Colitis UK have used the IBD Spotlight project in $2017 / 2018$ to support primary care physicians with the aim of improving detection and diagnosis of IBD. ${ }^{47}$ This campaign was conducted just after our study period and further research is needed to determine its impact.

\section{Conclusions}

There is a significant excess of GI symptoms 5 years before diagnosis of IBD compared to the background population which is likely attributable to undiagnosed disease. Previous diagnoses of IBS and depression are associated with a delay in specialist review. Enhanced pathways are needed to accelerate specialist referral and timely IBD diagnosis.

\section{Table 1: Baseline characteristics of study population}

\begin{tabular}{l|rrrr|}
\hline IBD Status & $\begin{array}{c}\text { Crohn's } \\
\text { Disease }\end{array}$ & \multicolumn{1}{c}{$\begin{array}{c}\text { Controls } \\
\text { (CD) }\end{array}$} & \multicolumn{1}{c}{$\begin{array}{c}\text { Ulcerative } \\
\text { Colitis }\end{array}$} & \multicolumn{1}{c|}{$\begin{array}{c}\text { Controls } \\
\text { (UC) }\end{array}$} \\
\hline $\mathbf{n =}$ & $\mathbf{5 , 8 7 4}$ & $\mathbf{2 3 , 4 3 6}$ & $\mathbf{1 3 , 6 8 1}$ & $\mathbf{5 4 , 6 1 6}$ \\
$\begin{array}{l}\text { Demographics } \\
\text { Male (\%) }\end{array}$ & $2,721(46)$ & $10,848(46)$ & $7,278(53)$ & $29,054(53)$ \\
\hline $\begin{array}{l}\text { Age at diagnosis (years) (\%) } \\
\text { (U) }\end{array}$ & & & & \\
\end{tabular}


$<17$

17-39

$>39$

Social deprivation (\%)

IMD 1-3

IMD 4-5

Unknown
$492(8)$

$2,420(41)$

$2,961(51)$

$1,961(8)$

$9,654(41)$

$380(3)$

$1,522(3)$

4,194 (31)

$9,106(67)$

$16,756(31)$

$11,817(51)$

2,187 (37)

$1,243(21)$

$8,466(36)$

$4,969(21)$

$5,692(42)$

2,437 (18)

20,332 (37)

$2,444(42)$

$10,001(43)$

Chronic GI symptoms - Individuals who presented twice within a 6 month period for gastrointestinal symptoms, where the presentations were at least 6 weeks apart

IMD - Index of Multiple Deprivation, 1 represents the least deprived and 5 represents the most deprived

Premorbid Depression - The patient had a previous diagnosis of depression prior to this presentation with chronic gastrointestinal symptoms

Premorbid IBS - The patient had a previous diagnosis of Irritable Bowel Syndrome prior to this presentation with chronic GI symptoms

Unattended Appointment - The patient was offered but did not attend a specialist appointment between the time of presenting to their GP with chronic Gl symptoms and finally attending a specialist appointment 
Table 2: Probability of specialist review in the 18 months following presentation with chronic GI symptoms to a primary care physician

Baseline Characteristics $\mathrm{n}=\mathbf{1 , 0 3 4}$

$$
\text { n (\%) }
$$

379 (37)

$592(57)$

590 (57)

444 (43)

$696(67)$

$338(33)$

1.12

574 (55)

165 (16)

295 (29)

174 (17)

173 (17)

Premorbid IBS

Era of presentation

Multiple Cox Regression $\mathrm{n}=\mathbf{1 , 0 3 4}$

HR 95\% CI

$0.46-1.01$

$0.78-1.13$

0.94

$0.86-1.23$

1.03

$0.93-1.35$

Smoking Status

Smoker

Ex-Smoker

Premorbid Depression

0.78

$0.79-1.33$

0.94-1.40

1.14

0.61-0.99

0.77

0.59-0.99 
2003-2006

2007-2010

2011-2013

2014-2016

Unattended Appointment
$473(46)$

$363(35)$

$165(16)$

$33(3)$

$143(14)$
1

2.07

1.67-2.56

3.89

8.53

3.06-4.95

5.78-12.6

$0.52-0.89$

All variables are included in the analysis. Results with a $p$-value of $<0.05$ are highlighted in bold.

Chronic Gl symptoms - Individuals who presented twice within a 6 month period for gastrointestinal symptoms, where the presentations were at least 6 weeks apart

IMD - Index of Multiple Deprivation, 1 represents the least deprived and 5 represents the most deprived

Premorbid Depression - The patient had a previous diagnosis of depression prior to this presentation with chronic gastrointestinal symptoms

Premorbid IBS - The patient had a previous diagnosis of Irritable Bowel Syndrome prior to this presentation with chronic $\mathrm{Gl}$ symptoms

Unattended Appointment - The patient was offered but did not attend a specialist appointment between the time of presenting to their GP with chronic GI symptoms and finally attending a specialist appointment 


\section{References}

1. Hong Z, Ren J, Li Y, et al. Delayed diagnosis is associated with early and emergency need for first crohn's disease-related intestinal surgery. Med Sci Monit. 2017;23:4841-4846. doi:10.12659/MSM.904238

2. Lee $D$, Koo JS, Choe JW, et al. Diagnostic delay in inflammatory bowel disease increases the risk of intestinal surgery. World J Gastroenterol. 2017;23(35):6474-6481. doi:10.3748/wjg.v23.i35.6474

3. Safroneeva E, Vavricka SR, Fournier N, et al. Impact of the early use of immunomodulators or TNF antagonists on bowel damage and surgery in Crohn's disease. Aliment Pharmacol Ther. 2015;42(8):977-989. doi:10.1111/apt.13363

4. Chhaya V, Pollok RCG, Cecil E, et al. Impact of early thiopurines on surgery in 2770 children and young people diagnosed with inflammatory bowel disease: A national population-based study. Aliment Pharmacol Ther. 2015;42(8):990-999. doi:10.1111/apt.13361

5. Magro F, Rodrigues-Pinto E, Coelho R, et al. Is it possible to change phenotype progression in crohn's disease in the era of immunomodulators? predictive factors of phenotype progression. Am J Gastroenterol. 2014;109(7):1026-1036. doi:10.1038/ajg.2014.97

6. Cosnes J, Bourrier A, Laharie D, et al. Early Administration of Azathioprine vs Conventional Management of Crohn's Disease: A Randomized Controlled Trial. 2013:758-765. doi:10.1053/j.gastro.2013.04.048

7. Aloi M, D’Arcangelo G, Bramuzzo M, et al. Effect of Early Versus Late Azathioprine Therapy in Pediatric Ulcerative Colitis. Inflamm Bowel Dis. 2016;22(7):1647-1654. doi:10.1097/MIB.0000000000000828

8. Probert CSJ, Dignass AU, Lindgren S, Oudkerk Pool M, Marteau P. Combined oral and rectal mesalazine for the treatment of mild-to-moderately active ulcerative colitis: rapid symptom 
resolution and improvements in quality of life. J Crohns Colitis. 2014;8(3):200-207. doi:10.1016/j.crohns.2013.08.007

9. Travis S, Feagan BG, Peyrin-Biroulet L, et al. Effect of Adalimumab on Clinical Outcomes and Health-related Quality of Life Among Patients With Ulcerative Colitis in a Clinical Practice Setting: Results From InspirADA. J Crohns Colitis. 2017;11(11):1317-1325. doi:10.1093/eccojcc/jjx093

10. Fuentes D, Torrente F, Keady S, et al. High-dose azathioprine in children with inflammatory bowel disease. Aliment Pharmacol Ther. 2003;17(7):913-921. doi:10.1046/j.13652036.2003.01540.x

11. Berg DR, Colombel J-F, Ungaro R. The Role of Early Biologic Therapy in Inflammatory Bowel Disease. Inflamm Bowel Dis. April 2019. doi:10.1093/ibd/izz059

12. Stapley SA, Rubin GP, Alsina D, Shephard EA, Rutter MD, Hamilton WT. Clinical features of bowel disease in patients aged $<50$ years in primary care: A large case-control study. $\mathrm{Br} J \mathrm{Gen}$ Pract. 2017;67(658):e336-e344. doi:10.3399/bjgp17X690425

13. Card TR, Siffledeen J, Fleming KM. Are IBD patients more likely to have a prior diagnosis of irritable bowel syndrome? Report of a case-control study in the General Practice Research Database. United Eur Gastroenterol J. 2014;2(6):505-512. doi:10.1177/2050640614554217

14. Cantoro L, Sabatino A Di, Papi C, et al. The time course of diagnostic delay in inflammatory bowel disease over the last sixty years: an Italian multicentre study. J Crohns Colitis. 2017;17(3):E-published ahead of print. doi:10.1093/ecco-jcc/jjx041

15. Vavricka SR, Spigaglia SM, Rogler G, et al. Systematic Evaluation of Risk Factors for Diagnostic Delay in. Inflamm Bowel Dis. 2012;18(3):496-505. doi:10.1002/ibd.21719

16. Lamb CA, Kennedy NA, Raine T, et al. British Society of Gastroenterology consensus 
guidelines on the management of inflammatory bowel disease in adults. Gut. 2019:gutjnl2019-318484. doi:10.1136/gutjnl-2019-318484

17. Kapasi R, Glatter J, Lamb CA, et al. Consensus standards of healthcare for adults and children with inflammatory bowel disease in the UK. Frontline Gastroenterol. 2019:flgastro-2019101260. doi:10.1136/flgastro-2019-101260

18. Herrett E, Gallagher AM, Bhaskaran K, et al. Data Resource Profile: Clinical Practice Research Datalink (CPRD). Int J Epidemiol. 2015;44(3):827-836. doi:10.1093/ije/dyv098

19. Lewis JD, Ms CB, Bilker WB, Strom BL. Validity and completeness of the General Practice Research Database for studies of inflammatory bowel disease. Pharmacoepidemiol Drug Saf. 2002;(April):211-218.

20. van Staal TP, Card T, Logan RF, et al. 5-Aminosalicylate use and colorectal cancer risk in inflammatory bowel disease: a large epidemiological study. Gut. 2005;54(11):1573-1578. doi:10.1136/gut.2005.070896

21. Armstrong RG, West J, Card TR. Risk of cancer in inflammatory bowel disease treated with azathioprine: a UK population-based case-control study. Am J Gastroenterol. 2010;105(7):1604-1609. doi:10.1038/ajg.2009.745

22. Blackwell J, Saxena S, Alexakis C, et al. The impact of smoking and smoking cessation on disease outcomes in ulcerative colitis: a nationwide population-based study. Aliment Pharmacol Ther. 2019;(March):1-12. doi:10.1111/apt.15390

23. Chhaya V, Pollok R. The impact of thiopurines on surgical outcomes in inflammatory bowel disease: do they make a difference? F1000Prime Rep. 2013;5(November):50. doi:10.12703/P5-50

24. Hoyer N, Prior TS, Bendstrup E, Wilcke T, Shaker SB. Risk factors for diagnostic delay in 
idiopathic pulmonary fibrosis. Respir Res. 2019;20(1):103. doi:10.1186/s12931-019-1076-0

25. Aires A, Barros A, Machado C, et al. Diagnostic Delay of Multiple Sclerosis in a Portuguese Population. Acta Med Port. 2019;32(4):289-294. doi:10.20344/amp.11187

26. Dianatinasab M, Mohammadianpanah M, Daneshi N, Zare-Bandamiri M, Rezaeianzadeh A, Fararouei M. Socioeconomic Factors, Health Behavior, and Late-Stage Diagnosis of Breast Cancer: Considering the Impact of Delay in Diagnosis. Clin Breast Cancer. 2018;18(3):239-245. doi:10.1016/j.clbc.2017.09.005

27. Kweon S-S, Kim M-G, Kang M-R, Shin M-H, Choi J-S. Difference of stage at cancer diagnosis by socioeconomic status for four target cancers of the National Cancer Screening Program in Korea: Results from the Gwangju and Jeonnam cancer registries. J Epidemiol. 2017;27(7):299304. doi:10.1016/J.JE.2016.07.004

28. Marston L, Carpenter JR, Walters KR, et al. Smoker, ex-smoker or non-smoker ? The validity of routinely recorded smoking status in UK primary care : a cross-sectional study. BMJ Open. 2014:1-7. doi:10.1136/bmjopen-2014-004958

29. Blackwell J, Saxena S, Pollok RC. Changing trends in coding for depression among the UK inflammatory bowel disease population. Gut. February 2019:gutjnl-2019-318296. doi:10.1136/gutjnl-2019-318296

30. Pimentel M, Chang M, Chow EJ, et al. Identification of A Prodromal Period in Crohn's Disease But Not Ulcerative Colitis. Am J Gastroenterol. 2000;95(12). https://journals.Iww.com/ajg/Fulltext/2000/12000/Identification_of_A_Prodromal_Period_in _Crohn_s.19.aspx.

31. Nguyen VQ, Jiang D, Hoffman SN, et al. Impact of Diagnostic Delay and Associated Factors on Clinical. Inflamm Bowel Dis. 2017;23(10):1825-1831. doi:10.1097/MIB.0000000000001257 
32. Henriksen M, Hoivik ML, Jelsness-Jorgensen L-P, Moum B. Irritable Bowel-like Symptoms in Ulcerative Colitis are as Common in Patients in Deep Remission as in Inflammation: Results From a Population-based Study [the IBSEN Study]. J Crohns Colitis. 2018;12(4):389-393. doi:10.1093/ecco-jcc/jjx152

33. Menees SB, Powell C, Kurlander J, Goel A, Chey WD. A Meta-Analysis of the Utility of CReactive Protein, Erythrocyte Sedimentation Rate, Fecal Calprotectin, and Fecal Lactoferrin to Exclude Inflammatory Bowel Disease in Adults With IBS. Am J Gastroenterol. 2015;110(3):444-454. http://dx.doi.org/10.1038/ajg.2015.6.

34. Danese S, Fiorino G, Mary JY, et al. Development of red flags index for early referral of adults with symptoms and signs suggestive of Crohn's disease: An IOIBD initiative. J Crohn's Colitis. 2015;9(8):601-606. doi:10.1093/ecco-jcc/jjv067

35. Gomollón F, Dignass A, Annese V, et al. 3rd European Evidence-based Consensus on the Diagnosis and Management of Crohn's Disease 2016: Part 1: Diagnosis and Medical Management on behalf of ECCO. J Crohn's Colitis. 2016:1-23. doi:10.1093/ecco-jcc/jjw168

36. Canavan C, Card T, West J. The incidence of other gastroenterological disease following diagnosis of irritable bowel syndrome in the UK: A cohort study. PLoS One. 2014;9(9). doi:10.1371/journal.pone.0106478

37. Barin L, Kamm CP, Salmen A, et al. How do patients enter the healthcare system after the first onset of multiple sclerosis symptoms? The influence of setting and physician specialty on speed of diagnosis. Mult Scler J. January 2019:1352458518823955. doi:10.1177/1352458518823955

38. Jones S, Howard L, Thornicroft G. 'Diagnostic overshadowing': worse physical health care for people with mental illness. Acta Psychiatr Scand. 2008;118(3):169-171. doi:10.1111/j.16000447.2008.01211.x 
39. Moscrop A, Siskind D, Stevens R. Mental health of young adult patients who do not attend appointments in primary care: A retrospective cohort study. Fam Pract. 2012;29(1):24-29. doi:10.1093/fampra/cmr053

40. Wang Y, Hunt K, Nazareth I, Freemantle N, Petersen I. Do men consult less than women ? An analysis of routinely collected UK general practice data. 2013:1-7. doi:10.1136/bmjopen2013-003320

41. Pellino G, Sciaudone G, Selvaggi F, Riegler G. Delayed diagnosis is influenced by the clinical pattern of Crohn's disease and affects treatment outcomes and quality of life in the long term: a cross-sectional study of 361 patients in Southern Italy. Eur J Gastroenterol Hepatol. $2015 ; 27(2)$. https://journals.Iww.com/eurojgh/Fulltext/2015/02000/Delayed_diagnosis_is_influenced_by _the_clinical.10.aspx.

42. Vadstrup K, Alulis S, Borsi A, et al. Cost Burden of Crohn's Disease and Ulcerative Colitis in the 10-Year Period Before Diagnosis-A Danish Register-Based Study From 2003-2015. Inflamm Bowel Dis. 2019;XX(Xx):1-6. doi:10.1093/ibd/izz265

43. Blackwell J, Saxena S, Pollok RC. Role of thiopurines as disease-modifying agents in Crohn's disease. Gut. January 2018. http://gut.bmj.com/content/early/2018/01/03/gutjnl-2017315840.abstract.

44. Markowitz J, Grancher K, Kohn N, Lesser M, Daum F. A multicenter trial of 6-mercaptopurine and prednisone in children with newly diagnosed Crohn's disease. Gastroenterology. 2000;119(4):895-902. doi:10.1053/gast.2000.18144

45. Fiorino G, Gilardi D, Bonovas S, et al. P244 Validation of the Red Flags index for early diagnosis of Crohn's disease: A prospective general practitioner study. J Crohn's Colitis. 2020;14(Supplement_1):S267-S269. doi:10.1093/ecco-jcc/jjz203.373 
46. Angelberger S, Vogelsang H, Novacek G, et al. Public awareness of Crohn's disease and ulcerative colitis: A national survey . J Crohn's Colitis. 2009;3(3):157-161. doi:10.1016/j.crohns.2009.01.003

47. Royal College of General Practitioners. IBD Spotlight Project.

https://www.rcgp.org.uk/clinical-and-research/our-programmes/clinical-priorities/spotlightprojects-2019-to-2020/inflammatory-bowel-disease.aspx. Published 2020. Accessed June 7, 2020. 
Figure 1
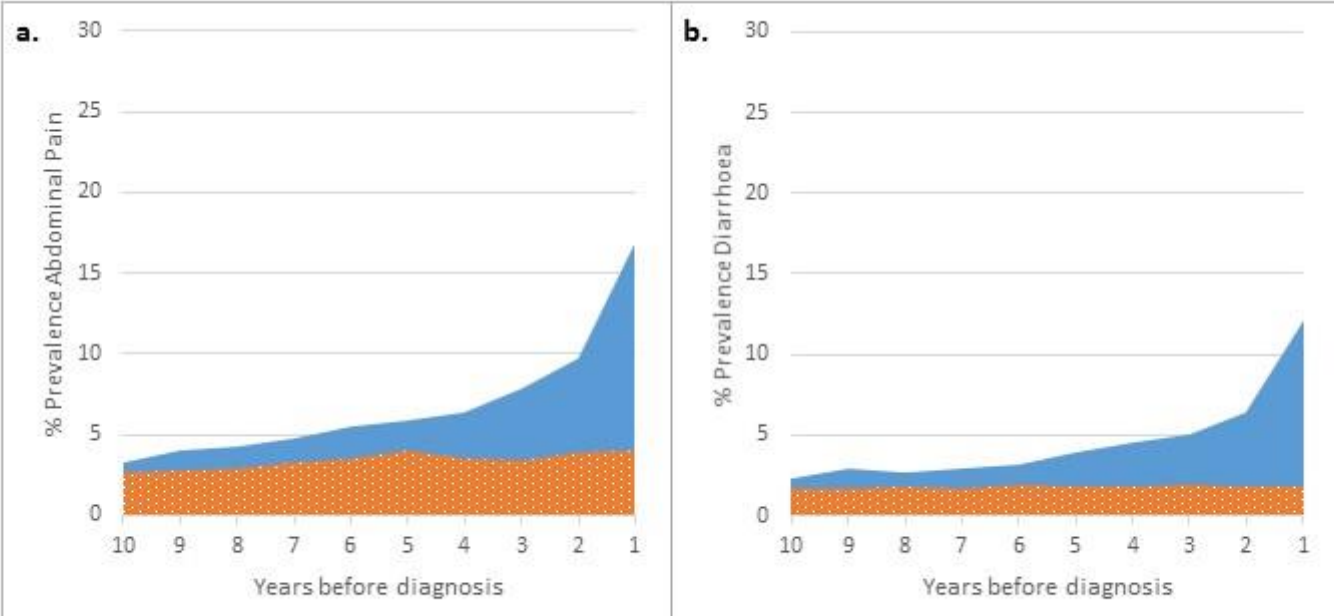

c. 30

d. 30
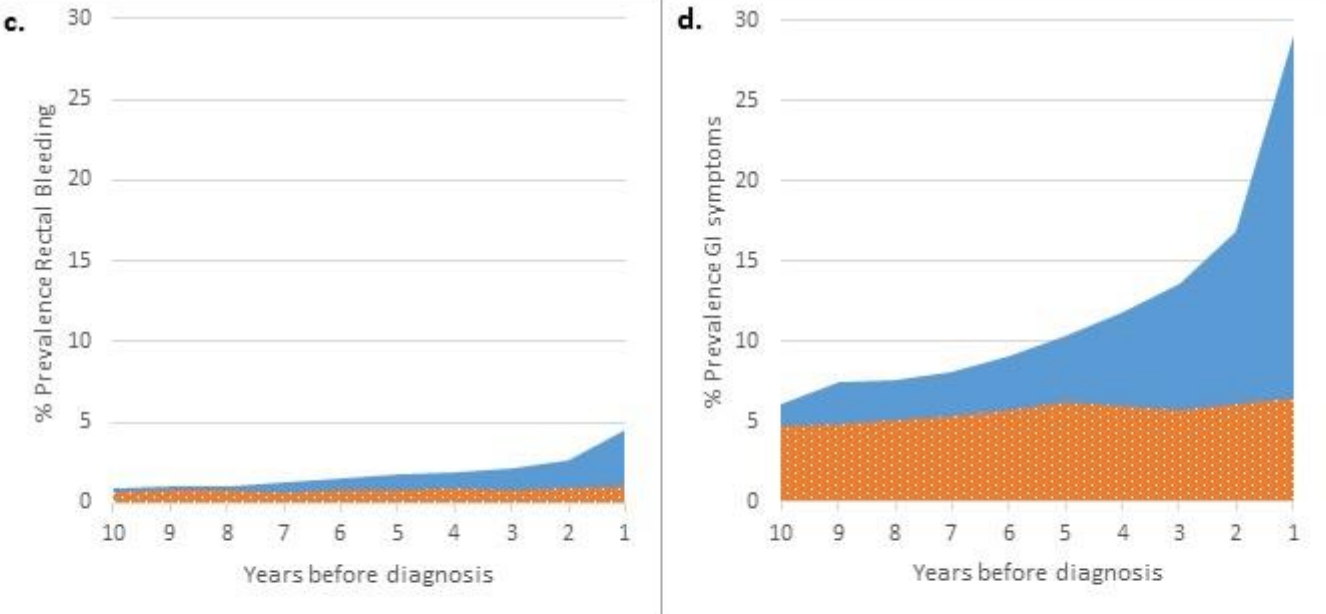

졸 Symptoms among Controls

Excess symptoms before Crohn's Disease 
Figure 2

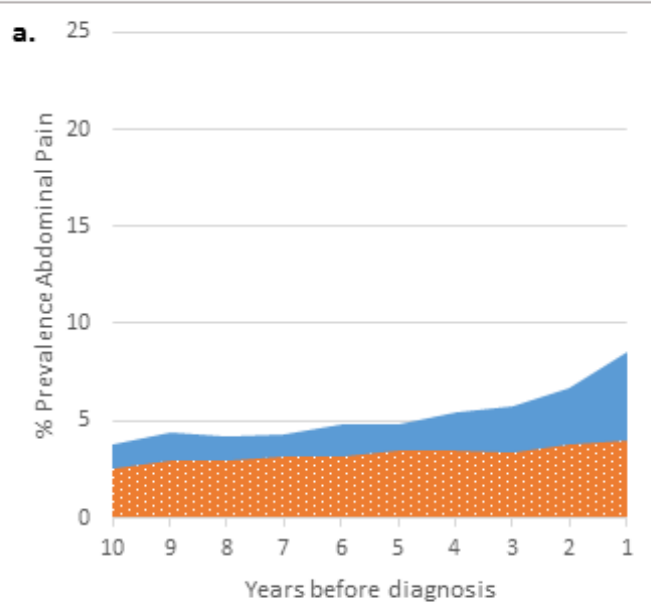

b. 25

c. 25

d. 25
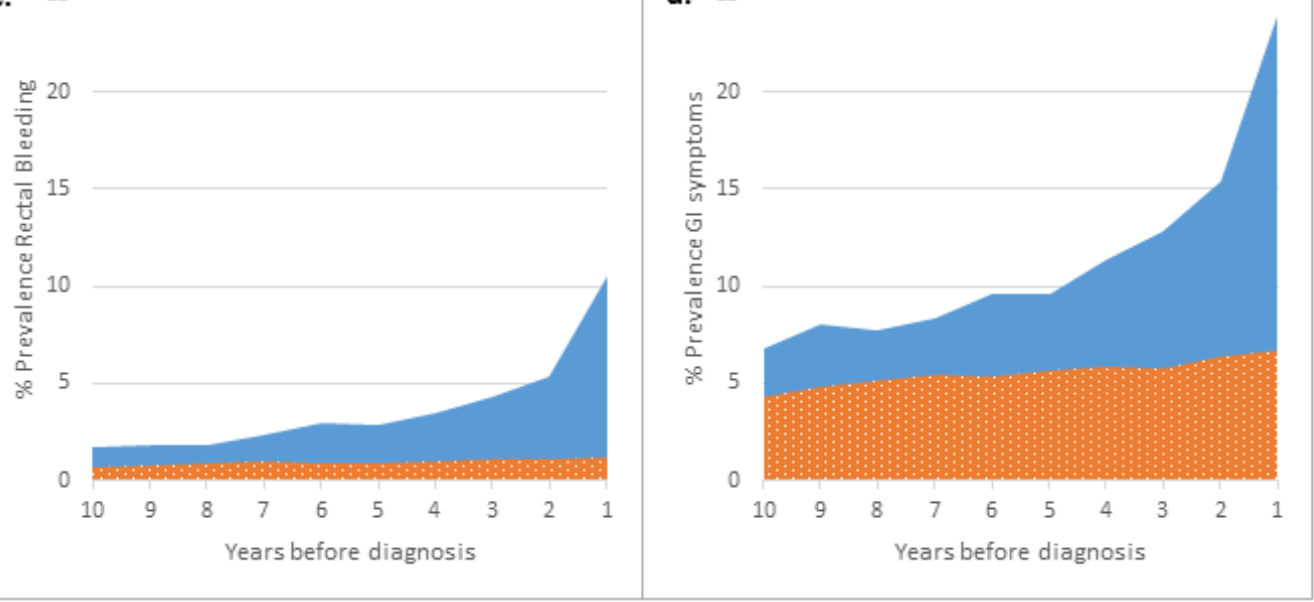

II Symptoms among Controls

Excess symptoms before ulcerative colitis 
Figure 3

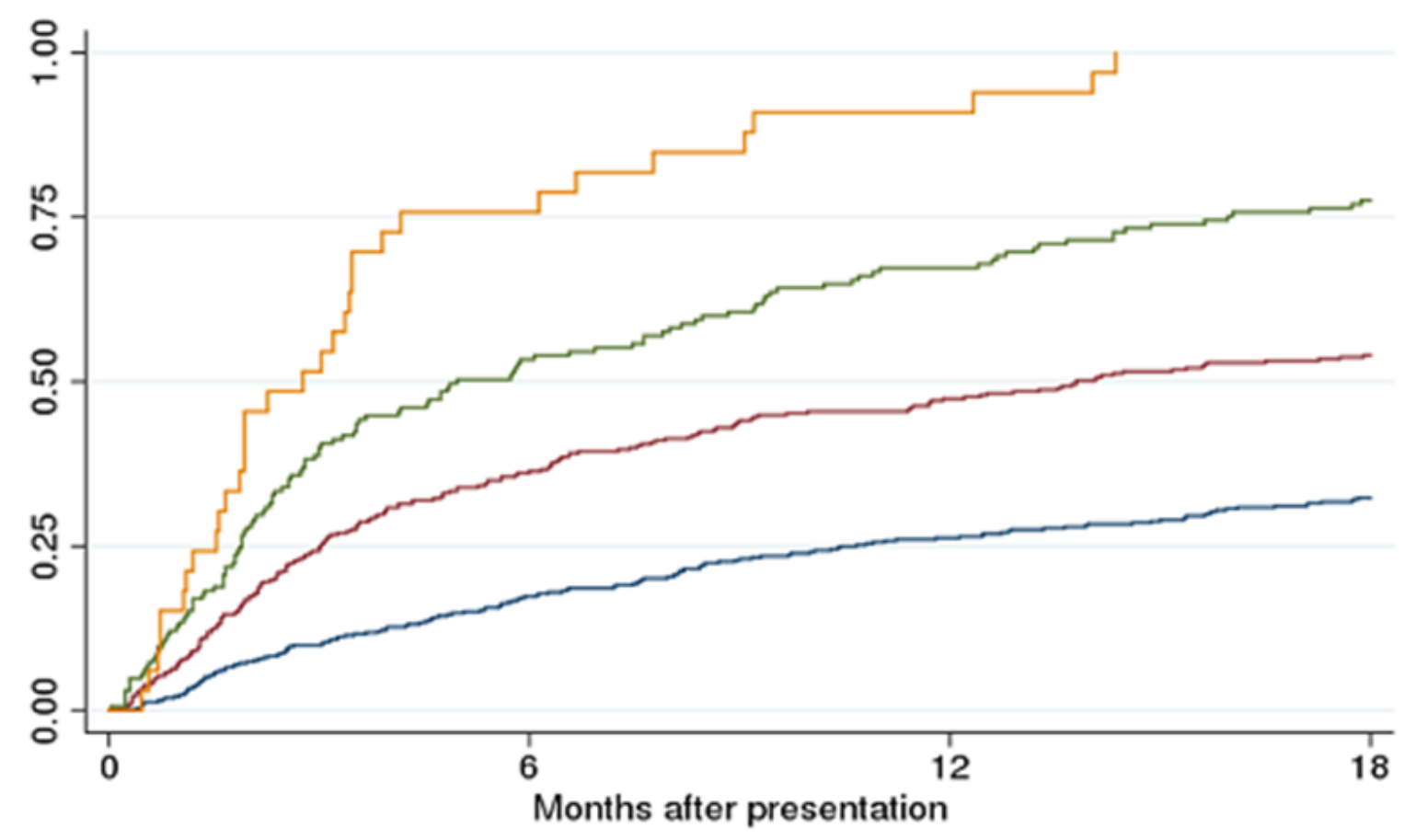


Manuscript Doi: 10.1093/ecco-jcc/jjaa146

Figure 4

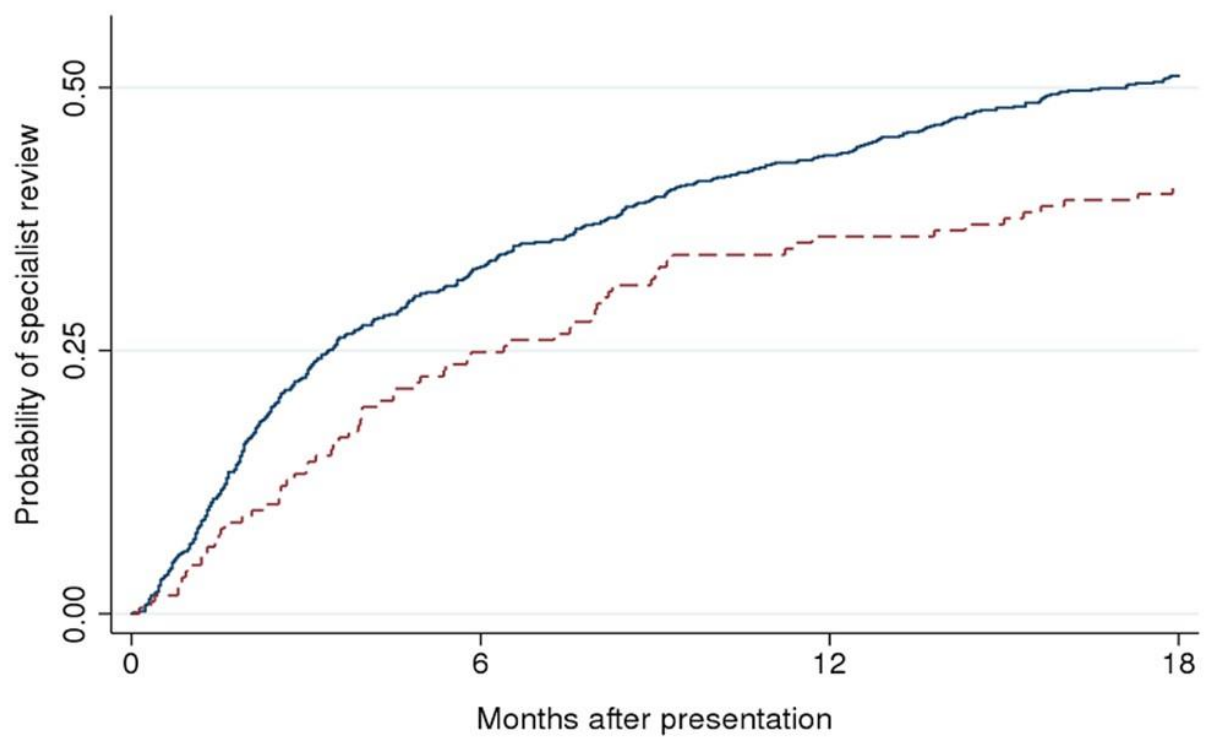

No IBS ----- Previous diagnosis of IBS 